\title{
Iranica nella Disputatio de Christo in Persia
}

\author{
Antonio Panaino \\ Università di Bologna
}

\begin{abstract}
This article insists on the importance of a very complex and intriguing Byzantine Greek text, usually denominated Narratio de rebus Persicis, De Gestis in Perside, or Disputatio de Christo in Persia, which contains a large amount of extremely interesting material enabling a better comprehension of the image of Iran in Western Late Antiquity from the point of view of the contemporary Christian perception. Among the main aims of this book, full of compositional strata of different origins and times (e.g. the very important and archaic prophesy of Jesus' birth through the voice of a star appeared to Cyrus the Great in a temple), one was certainly to criticise the extremely polemical attitude of opposing Christian Churches and communities in the East, particularly in the milieu of the Sasanian Empire. In fact, the great framework of the present work is occasionally offered by a theological debate, lasting for days, which should have been taken at the

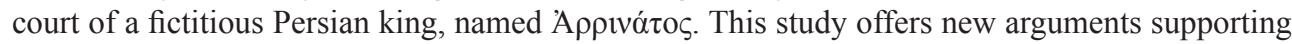
the presence in such a frequently forgotten Greek source of some clear references to the kingdoms of Kawād I and Xusraw I, with particular reference to anti-Mazdakite polemics. Furthermore, the final part of the article proposes an Iranological evaluation of the resonance produced by the name of the Persian protagonist of the whole book, the wise Aphroditianus (A
\end{abstract}

Key words: Narratio de rebus Persicis, De Gestis in Perside, Disputatio de Christo in Persia, anti-Mazdakite polemics, Aphroditianus, Kawād I, Xusraw I, Persia.

In uno studio recente ${ }^{1}$ ho cercato di portare l'attenzione della comunità scientifica orientalistica interessata ai rapporti tra Cristianesimo e Mazdeismo in età tardo-antica su di un testo molto particolare, che la tradizione umanistica ha in principio erroneamente attribuito a Giulio Africano (sotto il titolo di Africani Narratio de iis quae in Persia

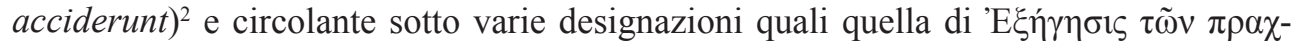

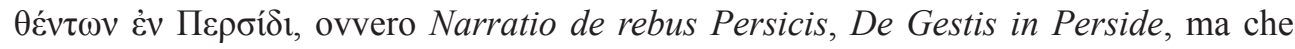
preferisco designare come Disputatio de Christo in Persia, titolo di riferimento che adotteremo per pura comodità. La storia ivi narrata si svolge presso la corte persiana di

\footnotetext{
1 Vedi Panaino 2011.

2 Vedi ancora Migne 1856, 97-107.
} 
epoca sasanide, in un'ambientazione che appare verisimilmente corrispondere a quella dell'epoca di Cosroe I, ovvero Xusraw Anōšag-ruwān (quindi, nel VI secolo d.C.), figura da intravedersi con ogni verosimiglianza dietro quella della persona del tutto fittizia del $\beta \alpha \sigma \imath \lambda \varepsilon v ́ \varsigma$ definito A $\rho \rho \imath v \alpha ́ \tau o \varsigma^{3}$

La vicenda occupa ben quattro diverse giornate piene di dispute e di controversie teologico-religiose; la prima coinvolge Pagani e Cristiani, impegnati in una violenta polemica a proposito degli oracoli che annuncerebbero la nascita di Cristo; la seconda descrive la sconfitta patita da uno stregone pagano di nome Oríkatos (indicato come "il primo degli incantatori" [ó $\pi \rho \tilde{\omega} \tau o \varsigma \tau \tilde{\omega} \nu \dot{\varepsilon} \pi \alpha 01 \delta \tilde{\omega} v])^{4}$ dinanzi ai Cristiani e fa soprattutto da introduzione al successivo ingresso nella concione degli Ebrei, irritati dall'eccessivo successo ottenuto dai Cristiani in presenza del loro comune sovrano. La terza e quarta giornata, infine, si concentrano sulla controversia tra Cristiani ed Ebrei, che si conclude con la conversione di una sessantina di Ebrei. Nell'economia del testo hanno, però, molto peso sia la cosiddetta "Storia di Cassandro" (che raccoglie diversi oracoli di origine pagana a supporto della nascita di Gesù), sia la "Narrazione di Aphroditianus", un anziano filosofo (che viene, infatti, indicato come ottantenne) di evidente cultura greco-persiana, ${ }^{5}$ il quale agisce nel corso delle successive controversie come arbitro. Egli, pur rappresentando implicitamente la tradizione persiana (e, forse, mazdea), ${ }^{6}$ mostra non solo di possedere una vasta competenza in cui cultura iranica ed ellenismo si affiancano, ma manifesta una notevole conoscenza del Cristianesimo, delle cui ragioni egli diventa nel corso delle discussioni un (apparentemente neutrale) araldo e difensore. Nel corso della stessa "Narrazione di Aphroditianus" (ovvero A A $\rho \circ \delta \imath \tau \imath \alpha v o ́ \varsigma))^{7}$ è, inoltre, ambientata l'accurata descrizione di un'importante leggenda cristiana, ben nota anche da altre fonti, in cui viene narrata una sorta di annunciazione ante litteram della natività del Cristo, anticipata di molti secoli rispetto alla sua futura nascita del Salvatore, perché rivelata con una serie di eventi miracolosi addirittura durante il regno di Ciro il Grande (VI sec. a.C.). Infatti, secondo tale tradizione, nel tempio di Héra, la cui costruzione viene attribuita proprio a Ciro, re di Persia, ${ }^{8}$ il Gran Sacerdote avrebbe annunziato al sovrano il futuro parto della dea Héra, "Celeste" (Oủpaví $\alpha){ }^{9}$ dopo la felice unione con il Grande

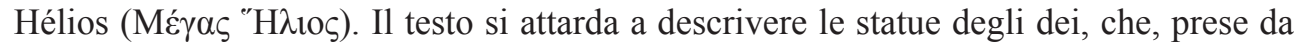

3 Bratke 1899, passim.

${ }^{4}$ Cfr. Bratke 1899, 22. Si deve notare il fatto che l'anonimo autore non fa deliberatamente uso del termine $\mu \alpha \gamma_{\gamma} \varsigma$, per evitare un'indebita confusione con i Magi recatisi a Betlemme.

5 Heyden 2009, 2, lo considera chiaramente "persiano". Cfr. Bringel 2007, 44-46, passim.

6 Si noti, però, che la sua figura richiama anche quella di Daniele, come nota Eastbourne 2011, 3. Aphroditianus è esplicitamente connesso alla sapienza dei Caldei e dei Babilonesi (Bratke 1899, 3, 27-28). Daniele è, invece, ancora menzionato in 30, 11 (ed. Bratke). Si ricordi che quando il re (probabilmente Ciro) entra nel tempio di Hera $(11,14$, ed. Bratke), egli intende ottenere un'interpretazione di sogni. Il contesto richiama, pertanto, quello del Libro di Daniele, ove gli indovini, specialisti di oneiromanzia, corrispondono ai magupersiani, secondo la versione dei Septuaginta. Vedi Panaino 2004a, 12-25.

7 Cfr. la voce Aphroditianos, a cura di Schwartz 1894, 2788-2793. Heyden (2009, 1) precisa che tale filosofo sarebbe sostanzialmente sconosciuto, ma nota, pur con tutti i dubbi del caso, il fatto che un certo Adfroditianus compare nella Ravennatis anonymi Cosmographia (ed. Schnetz 1940, 22, 52-55).

${ }^{8}$ La descrizione del tempio fatto costruire da Ciro potrebbe essere stata influenzata dalla tradizione appartenente al Romanzo di Alessandro dello Pseudo-Callistene; questa è la teoria proposta da Kampers (1901, 116-135), su cui però si vedano le prudenti considerazioni della Heyden 2009, 61, 262 e soprattutto 271-275.

${ }^{9}$ Cfr. Heyden 2009: 15 e passim. 
infinita contentezza per tale evento, avrebbero tutte cominciato a danzare. ${ }^{10}$ La dea, in

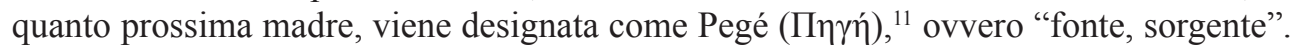
Appare evidente il riferimento sempre più esplicito a Maria, dato che il testo afferma che la dea avrebbe sposato un "carpentiere". Risulta ancor più interessante notare come il fatto di essere "fonte garantirebbe lo scorrere perenne dello Spirito, attraverso cui un solo pesce (ovvero lo stesso Gesù) ${ }^{12}$ può procedere". Sulla scena appare finalmente anche una stella di particolare splendore, che discende dal cielo, per fermarsi proprio sulla statua della dea "Fonte", onde rivelare al sovrano persiano la futura nascita verginale di un bambino straordinario, definito come "il Principio della salvezza e la Fine della distruzione". Questo bambino sarebbe il figlio del grande Sole (Hélios), ma inteso come una divinità dotata di tre nomi $\left(\tau \rho \iota \omega_{v} \nu \mu \rho \varsigma\right),{ }^{13}$ designazione da cui si evince un chiarissimo riferimento trinitario. A quel punto tutte le statue, con l'eccezione di quella su cui l'astro si era fermato, si sgretolano. Non deve sfuggire il fatto che la stella non solo si lascia distinguere per l'eccezionale luminosità, ma anche per il possesso di un "diadema

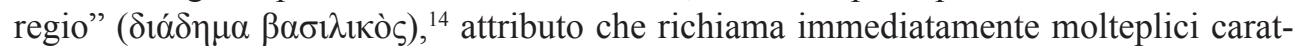
teristiche della regalità iranica ed ellenistica. Una voce celeste ordina a Ciro di inviare i suoi Magi a Gerusalemme, mentre la stella rimane stazionaria sulla statua sino alla partenza del corteo dei Magi persiani. Compare anche il dio Dioniso, al quale si deve la predizione secondo cui lo straordinario fanciullo avrebbe scacciato tutti i falsi dei; sempre Dioniso dichiara che Pegé non sarebbe più stata figura umana, bensì sovrumana, per aver concepito un essere generato dalla divina Fortuna (Tú $\chi) ;{ }^{15}$ quest'ultimo richiamo alla "Fortuna regia" evoca immediatamente una serie di temi iranici strettamente connessi alla sfera della regalità e del sacerdozio, la cui rilevanza è oltremodo significativa in contesto tardo antico tra Bisanzio e l'Iran. Secondo la stessa narrazione, allora, i Magi, ${ }^{16}$ si sarebbero recati a Betlemme sulla scia della stella per rendere omaggio a Gesù e Maria, che avrebbero al fine trovato, come avviene secondo una ben consolidata tradizione della Natività, solo dopo l'incontro con Erode e la sua corte. Si deve altresì osservare che secondo il racconto di Aphroditianus gli emissari di Erode avrebbero tentato di corrompere tali Magi, offrendo loro ricchi doni ${ }^{17}$ in cambio del silenzio sulla prossima nascita del Salvatore e del loro immediato rientro in patria. La risposta dei Magi avrebbe fatto, a sua volta, leva sul ricordo minaccioso della cattività babilonese, intesa ora come punizione per l'empietà mostrata dagli Ebrei dinanzi all'evidenza della rivelazione. Quando i Magi giungono infine da Gesù, essi lo trovano in piedi, ma non in grembo alla madre, bensì per terra, secondo un'iconografia talmente desueta che parrebbe aver provocato non poco imbarazzo tanto nel corso della tradizione manoscritta di quest'opera quanto

${ }^{10}$ Sull'animazione delle statue, attestata in Siria ed Egitto, cfr. Bringel 2007, 46.

11 Vedi Kaufmann 1901, 529-548; cfr. Bringel 2007, 264-267, 332-333, passim.

12 Sull'immagine del "pesce" in questo testo, si veda Heyden 2009, 243-245 e passim. Cfr. Dölger 1922. Cfr. Bringel 2007, 266-267, 335-336.

${ }_{13}$ Bratke 1899, 13, 17.

${ }_{14}$ Bratke 1899, 13, 21 e 14, 15; Bringel 2007, 268-269, 340-343.

${ }_{15}$ Bratke 1899, 14, 18; 21, 21; 202, 203; Bringel 2007, 268-269, 244-245.

16 Bringel 2007, 47-48.

${ }^{17}$ Cfr. Bringel 2007, 270-273, 352-357. Per quanto concerne invece le questione dei doni recati dai Magi e le differenti versioni attestate nella tradizione manoscritta si veda ancora la Bringel 2007, 50-52, 272-273, 364-365. 
sul piano della rappresentazione artistica. ${ }^{18}$ Gli stessi Magi avrebbero poi fatto dipingere il ritratto di Gesù, ${ }^{19}$ come un fanciullo dell'età di circa due anni, ${ }^{20}$ nonché quello di sua

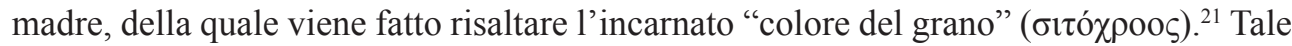
ritratto, una volta riportato in Persia presso il loro tempio più importante, sarebbe stato arricchito dal testo di un'iscrizione molto interessante:

Nel tempio divinamente fondato, ${ }^{22} \alpha$ Zeus Hélios, Dio Grande, re, Gesù, l'Impero Persiano dedicò

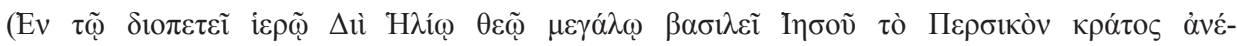
$\theta \eta \kappa \varepsilon v){ }^{23}$

Solo la trattazione di questo ritratto e della Vorlage letteraria riguardante tale parte del testo ci bloccherebbe a lungo, visto che si tratta di un passo fortemente risonante con quanto già si narra anche nella Dottrina di Addai, ${ }^{24}$ a proposito di un ritratto miracoloso che avrebbe fatto guarire re Abgar. Sul piano storico, inoltre, la Bringel si è legittimamente interrogata ${ }^{25}$ sul fatto che la storia del ritratto potrebbe aver tratto in qualche modo ulteriore ispirazione da quella tradizione greca, recepita da Evagrio Scolastico (IV.27), secondo la quale la sacra immagine avrebbe protetto la città di Edessa durante l'assedio posto proprio da Cosroe I nel 544. In ogni caso, anche tale questione si muove nuovamente attorno al periodo di Cosroe dall'Anima Immortale.

Non è mio scopo soffermarmi sui maggiori problemi di composizione del testo, che sono particolarmente complessi e che richiedono competenze specifiche, ma solo fare un breve e doveroso accenno a tale problematica. Secondo la Bringel, ${ }^{26}$ che ha dedicato nel 2007 una tesi dottorale a tale opera, tesi in cui è stata proposta una prima revisione radicale dell'edizione critica di riferimento, tuttora fondamentale, redatta e commentata da Bratke, ${ }^{27}$ il testo si presenterebbe in due diverse varietà, delle quali la stessa Bringel ha proposto un'edizione: una versione breve, molto più coerente e meno intrisa di antigiudaismo, ${ }^{28}$ ed una versione lunga, apparentemente recenziore. In una successiva revisione del suo studio, ${ }^{29}$ la stessa studiosa ha però ritenuto che gli argomenti volti a sostenere l'esistenza di una recensione breve mancherebbero di sufficiente solidità. Di grande interesse anche l'approccio della Heyden, ${ }^{30}$ la quale, pur senza purtroppo tenere in conto il lavoro della Bringel, ha messo in evidenza la presenza in questa fonte etero-

${ }^{18}$ Bringel 2007, 54-61, 361-362.

19 Cfr. Bacci 2000, 33-34. Sulla questione del ritratto di Cristo nella letteratura contemporanea, anche in rapporto al prestigio di diversi santuari, si veda ancora Bringel 2007, 54-55.

${ }^{20}$ Cfr. Heyden 2009, 3 e passim.

${ }^{21}$ Bratke 1899, 17, 22, 161, 235-236.

22 Sulla cristianizzazione di tale composto, in origine significante "caduto dal cielo, inviato da Zeus", si veda la Bringel nella nota 70 all'edizione on line.

${ }_{23}$ Bratke 1899, 18, 3-4; Heyden 2009, 16; Bringel 2007, 274-275, 360-363.

${ }^{24}$ Cfr. Desreumaux 1993, 59.

25 Vedi http://ctesiphon.huma-num.fr/sources-litteraires/intro-d/de-gestis-in-perside/\#_ftn73.

26 Bringel 2007, passim.

27 Bratke 1899, passim.

${ }^{28}$ Cfr. Bringel 2007, 62-72.

29 Vedi Bringel, http://ctesiphon.huma-num.fr/sources-litteraires/intro-d/de-gestis-in-perside/\#_ftn73, alla nota 2 .

30 Heyden, 2009, 13-18, passim. 
genea e complessa di un tema centrale, che si articola secondo almeno cinque differenti varietà narrative. Per quanto concerne poi la paternità della Disputatio, che è stata alternativamente attribuita, a seconda dei codici e delle differenti tradizioni, ad autori diversi, la questione, che in questa sede non riprendiamo, resta ancora aperta. ${ }^{31}$ A complicarne lo studio, ma anche a confermarne l'importanza, si aggiunge la presenza di numerose redazioni della Disputatio in antico-slavo e nella varietà slavo-meridionale, ${ }^{32}$ in rumeno, nonché, forse, addirittura di una versione armena. ${ }^{33}$ Rispetto alle ricerche precedenti, la Bringe $^{34}$ ha posto in modo più articolato la questione della datazione dell'opera. Così si noterà che la "Storia di Cassandro" e la "Storia di Aphroditianus" sono state tradizionalmente attribuite (o comunque messe in relazione) a Filippo di Side (in Panfilia), ${ }^{35}$ visto che occorrono anche nella sua Storia cristiana, databile tra il 425 ed il 439, mentre nella cosiddetta "recensione lunga" risulta interessante la menzione esplicita di un proconsole di Palestina, fatto che permetterebbe, come suggeriva sempre la Bringel, ${ }^{36}$ di fissare il terminus post quem al 536, data della Novella 103 di Giustiniano sulla riorganizzazione di quella regione. ${ }^{37}$ In ogni caso, come avevo già fatto notare in precedenza, il materiale compositivo di tale fonte (in particolare la "Storia dei Magi") sembrerebbe risalire ad un'epoca collocabile, come limite estremo inferiore, tra la fine del IV (meglio l'inizio del V) ed il VI secolo d.C., mentre il capitolo narrativo posto in bocca al filosofo Aphroditianus rivelerebbe un certo influsso da parte di Filippo di Side, ${ }^{38}$ celebre, peraltro, proprio per le sue polemiche contro Giuliano l'Apostata. Tali sarebbero, almeno in parte, alcune delle principali conclusioni tratte dalla Bringel (al momento della redazione della sua tesi), ${ }^{39}$ la quale, peraltro, propenderebbe, con molta prudenza, per una datazione tendenzialmente tardiva dell'opera nella sua completezza, da collocarsi tra la fine del VI ed il VII secolo. Si attende, comunque, che la studiosa francese pubblichi finalmente il suo lavoro in una versione definitiva.

Nel presente contributo non intendo più trattare della figura di Ciro, la cui presenza, come mi permetto solo di accennare, si inserisce in un ampio filone narrativo relativo alla Natività di Gesù in cui viene evocata la translatio imperii, dal Messia terrestre (Ciro come "unto del Signore) al Messia celeste, né della spesso malintesa questione dello scarto cronologico tra Ciro e Gesù, che sono invece associati non sull'asse della sincronia storica, del tutto impossibile ed assurda, ma dalla cifra distintiva della comune funzione "messianica" che trascende il tempo profano e che permette all'uno di inviare anzitempo i suoi emissari, i Magi, all'altro sovrano, in modo da riconoscere e sancire la superiore grandezza del divino successore. ${ }^{40}$ Per tali ragioni non entrerò nel merito di questi ed altri temi che coinvolgono il ciclo specifico dei Magi, a cui ho specificatamente dedicato numerosi lavori, mentre mi soffermerò su una serie di ulteriori elementi iranici

\footnotetext{
31 Vedi ancora Panaino 2011.

32 Vedi Bratke 1899, 50, 128; Bringel 2007, 123-127, 128-131.

33 Vedi Bratke 1899, 128. Cfr. Heyden 2009, passim.

34 Bringel 2007, 2-5, passim.

35 Cfr. Heyden 2006, 209-243, 171-225. Cfr. Bringel 2007, 40-41.

36 Bringel 2007, 2-5, 15-17, 20-25, passim.

37 Mayerson 1994, 294-300.

38 Vedi Honigmann 1953, 82-91; cfr. anche Monneret de Villard 1952, 108.

39 Bringel, 2007, 227-228.

40 Su tutto ciò si veda il mio studio del 2011 e gli altri lavori specifici ivi citati.
} 
propri di questa specifica fonte, fatta eccezione per i cosiddetti Persica, ovvero per quella serie di brevi passi (sette in totale) ${ }^{41}$ inseriti nel testo greco, che vorrebbero rappresentare vere e proprie frasi in (medio)-persiano, la cui discussione analitica richiederebbe una trattazione separata e di accurato dettaglio linguistico-filologico. Ci limiteremo così solo a notare che, purtroppo, né i tentativi esperiti da Joseph Marquart su richiesta di Bratke, ${ }^{42}$ né quelli successivamente messi in atto da Philip Huyse su invito della Bringel, hanno portato ad alcun riscontro realmente positivo; per il momento non posso che associarmi a tali rassegnate conclusioni, anche se bisogna notare che la presenza di lessemi o addirittura di stringhe di testo in pseudo-medio-persiano (anche se reso in alfabeto greco) e apparentemente indecifrabili (a parte qualche forma di vaga risonanza grecanica) indica, comunque, un forte desiderio di movimentare, anche sul piano fonosimbolico, il richiamo continuo all'ambientazione sasanide, se non altro attraverso il ricorso ad un esotismo di fantasia.

Tornando, invece, alla questione degli aspetti più interessanti dal punto di vista iranologico ricorrenti in quest'opera, mi preme, innanzitutto, evitare di smarrire lo sguardo d'insieme che da solo, invece, ci permette di osservare nella sua completezza come una fonte cristiana e bizantina tardo-antica deliberatamente ambienti la sua narrazione alla corte di un sovrano persiano, come abbiamo già visto, da indentificarsi con Cosroe I, per poi inglobarvi addirittura una storia che si svolge, invece, nel regno di Ciro il Grande. Probabilmente, l'atmosfera che tale testo rievoca risente più o meno direttamente dell'impressione prodotta dall'emigrazione di una nutrito numero di filosofi pagani alla corte ${ }^{43}$ proprio del sovrano di Persia Cosroe I dopo la chiusura delle scuole filosofiche di Atene, soprattutto dell'Accademia, avvenuta sotto il regno di Giustiniano nel 529. Non devono essere mancati anche ulteriori spunti positivi che venivano dai risultati conseguiti con la "Pace perenne" stipulata al termine della Guerra Lazika, che prevedeva addirittura uno specifico Addendum al trattato conclusivo concernente le minoranze religiose e che Menandro Scolastico (frag. 6, 1) (4 $^{44}$ presenta, per il pubblico di lingua greca, come particolarmente favorevole alla parte cristiana. ${ }^{45}$ Di fatto, gli elementi politicoculturali che si lasciano evincere in rapporto a tale opera sono molti: il mondo sasanide, almeno quello dell'epoca di Cosroe I, appare come un contesto capace di ospitare libere controversie teologiche, anzi una "piazza", diremmo noi oggi, in cui portare il Cristianesimo e da utilizzare non soltanto in funzione anti-pagana, ma soprattutto in un quadro che via via diviene sempre più anti-giudaico. ${ }^{46}$ Infatti, la Disputatio, a parte la vicenda dello stregone, ambientata nella seconda giornata, non si occupa molto di paganesimo, né aggredisce in modo particolarmente evidente la tradizione mazdaica. Se mai questa viene fatta oggetto di allusioni, più o meno dirette, nella narrazione di Aphroditianus a proposito della Natività di Gesù, ma si tratta di una porzione di testo che probabilmente

${ }^{41}$ Si veda la discussione della Bringel 2007, nonché quella proposta da Bratke 1899.

42 Bratke 1899, 248.

43 Vedi Frye 1983, 161; Panaino 2001.

${ }^{44}$ Testo secondo Blockley 1985, 54 (traduzione a p. 75). Per ulteriori note e discussioni su tale fonte vedi Panaino 2009; 2011/12.

45 Vedi Panaino 2009; 2011/12.

${ }^{46}$ Sul contesto geo-politico e culturale del regno di Xusraw I, si veda ora il ricco volume miscellaneo curato da Christelle Jullien (2015), che raccoglie una serie di interventi molto importanti ed aggiornati. 
appartiene ad una tradizione autonoma ancora più antica, la quale nuovamente presenta il mondo persiano, sebbene nella sua varietà achemenide, come parte integrante della storia della rivelazione del Cristo. La messa in scena di una struttura narrativa in cui un dotto persiano narra al suo sovrano (un re sasanide, alias Cosroe) una vicenda che si ambienta all'epoca del Gran Re Ciro (un achemenide) e che crea un legame ideologico-teleologico con un evento (la nascita di Cristo) che si sarebbe realizzato in epoca romanopartica, implica una sottile stesura cronologica e ciò forse concorre a spiegare le ragioni che potrebbero aver indotto alcuni copisti ad attribuire proprio a Julius Africanus tale opera. Per quanto siano aperte e spinose le discussioni tra gli storici dell'Iran a proposito della conoscenza o meno della storia del Casato achemenide in epoca sasanide, è certo che per i loro antagonisti occidentali il quadro delle successioni dinastiche della Persia antica non era affatto oscuro e che tale competenza non poteva che essere pienamente condivisa anche dalle Chiese orientali operanti all'interno dei confini dell'Impero Sasanide. In questo senso, la Disputatio conferma come l'area iranica sia stata oggetto di interesse potenzialmente missionario e considerata come terreno fertile per una possibile, anche se non facile, futura evangelizzazione.

Tra le diverse questioni più di dettaglio che concernono la materia iranologica in questa fonte vorrei, quindi, affrontarne nella presente occasione solo alcune in modo più specifico. Nel corso di uno dei diversi interventi di Aphroditianus, il saggio persiano

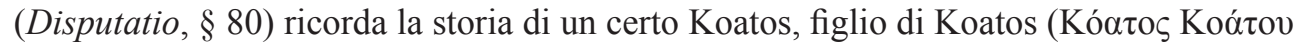
$\pi \alpha i \tilde{\zeta})$, che si sarebbe invaghito di una pastorella del villaggio di Arigbanes. Costei avrebbe però risolutamente rifiutato le avances del suo spasimante sino a preferire la morte per fame, dopo essere stata rinchiusa senza cibo da tale potente pretendente. In tal modo, ella avrebbe preservato tanto la sua verginità, quanto la libertà di unirsi al suo vero promesso sposo, riferimento che sembra alludere ad una auto-consacrazione allo stesso Cristo. Già il Wirth ${ }^{47}$ aveva supposto l'identificazione di tale Koatos con il sovrano Kawād, ma la Bringel ${ }^{48}$ ha sollevato una serie di obiezioni, in realtà molto fragili, su tale soluzione. Ella, infatti, si domanda:

S'agit-il de Kawadh I ${ }^{\text {er }}$ (488-531) ou de Kawadh II (628) ? Le court règne du second semble se situer à une date trop tardive, après les premières victoires d'Héraclius, pour avoir trouvé un écho dans un texte qui donne de la Perse une image si positive. Le premier fit exécuter son fils, mais dans des conditions fort différentes de notre exemple ascétique, dans lequel le père du jeune homme condamne ce dernier à être pendu lorsqu'il apprend que son fils a laissé périr de faim la bergère qui avait refusé de l'épouser, préférant mourir et ainsi « rendre intact» à son " pur fiancé » ce qui lui avait été donné, «la virginité, le pardon du mal, une existence sans richesse ... ». Il se peut cependant que le nom de Kawadh ait été repris dans la littérature du VI siècle.

Cerchiamo, innanzitutto, di ricostruire il contesto generale persiano che potrebbe aver ispirato l'anonimo compositore cristiano. L'episodio relativo all'assassinio di un figlio di Kawād I da parte dello stesso padre, a cui fa riferimento la Bringel, è del tutto aleatorio, essendo, infatti, stato Cosroe I a far uccidere non solo suo zio Bawi nel 531 a.C., colpevole di voler portare sul trono un altro membro della famiglia, ${ }^{49}$ ovvero il figlio di Zamāsp, anch'esso di nome Kawād; tale Zamāsp era, infatti, il secondo genito di Kawād I (suoi

\footnotetext{
47 Wirth 1894, 197.

48 Vedi http://ctesiphon.huma-num.fr/sources-litteraires/intro-d/de-gestis-in-perside/\#_ftn73.

49 Pourshariati 2008, 111, 268, 471.
} 
fratelli furono, infatti, nell'ordine, Kāwūs e Zāmāsp). ${ }^{50}$ La sorte di questo nobile più gio-

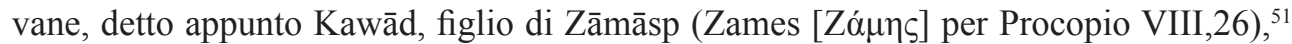

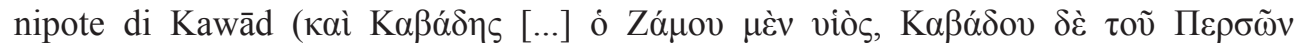

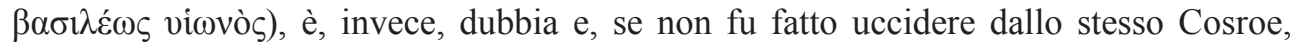
sembrerebbe aver cercato rifugio, dopo alcune vicende avventurose, nell'Impero Bizantino. ${ }^{52}$ Cosroe I, comunque fece eliminare tutti i suoi fratelli nello stesso contesto politico. ${ }^{53}$ In ogni caso, di tali omicidi il sovrano Kawād I restò del tutto estraneo (essendo, peraltro, già morto all'epoca di tali eventi). Al contrario, si deve ricordare che questo re in particolare si era, invece, mostrato estremamente generoso verso i suoi congiunti, soprattutto con il fratello Zāmāsp, nonostante il fatto che costui avesse regnato al suo posto negli anni 496-498, dopo che lo stesso Kawād era stato detronizzato per via del suo intricato rapporto con movimento mazdakita. ${ }^{54}$ Zāmāsp venne, infatti, lasciato sopravvivere contro ogni aspettativa, così come, d'altra parte, era accaduto allo stesso Kawād al momento della sua caduta dal trono.

A mio avviso, invece, la storia narrata da Aphroditianus si attaglia perfettamente ad un periodo che si snoda tra l'epoca di Kawād I e quella del figlio Cosroe; ad esempio, la vicenda dell'aggressione sessuale perpetrata contro la povera pastorella si inserisce molto bene nella rappresentazione, chiaramente distorta e svilita, dell'eresia mazdakita, ${ }^{55}$ presentata anche dalle fonti bizantine come portatrice non solo di un grave sconvolgimento sociale, ma anche di costumi sessualmente scabrosi, ${ }^{56}$ al punto da essere spesso rappresentata come una sorta di "comunismo delle donne". I rapporti, per quanto mutevoli, del sovrano Kawād I con Mazdak ed i suoi seguaci, ${ }^{57}$ ampiamente recepiti dalla letteratura bizantina, ${ }^{58}$ possono facilmente spiegare l'adattamento favolistico della vicenda narrata da Aphroditianus, in cui un potente signore (che si tratti di figura autorevole si evince dal fatto che egli può far imprigionare la fanciulla e farla morire di fame) di nome Koatos, figlio di Koatos (nome facilmente riconduci-

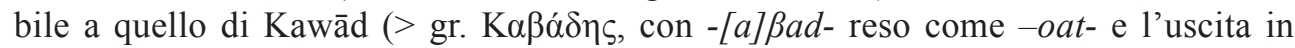
-o $\varsigma$ anziché in $-\eta \varsigma,{ }^{59}$ ma anche introdotto conformemente alla regola persiana dell'indicazione del patronimico), si macchia di un peccato di natura sessuale, che scaturisce dal desiderio irrefrenabile di possedere una fanciulla la quale, invece, gli si rifiuta.

${ }^{50}$ Pourshariati 2008, 288.

51 Cfr. ancora Gariboldi 2009, 112, nota 78.

52 Börm 2007, 54, 139, 256;.

53 Börm 2007, 104; Pourshariati 2008, 111-112; Gariboldi 2009, 109-113.

${ }^{54}$ Già il Wirth $(1894,197)$ menzionava Mazdak. in rapporto al sovrano Kawād I, ma non sviluppava alcuna ulteriore considerazione in proposito. Si veda la sintesi in Gariboldi 2009, 85-142.

55 Su Mazdakismo si rimanda alle trattazioni di Christensen 1925; Crone 1991; 2014; Gnoli 2004; Klíma 1957; 1977; Yarshater 1983; Wiesehöfer 2009.

56 Vedi Procopio I,5; cfr. Christensen 1925, 9.

57 Frye 1983, 151.

58 Se veda sul tema l'intervento del compianto amico e collega Fiaccadori 2006, che, nonostante la discutibile rilevanza attribuita al ruolo di Bundos, secondo una già nota proposta interpretativa di Pugliese Carratelli, offre la più aggiornata presentazione relativa alla ricezione dei temi mazdakiti a Bisanzio.

59 Si noti che epigraficamente il nome di Kawād corrisponde al pahlavi kw'ty e che, pertanto, una trascrizione come Koat-os appare formalmente perfetta, essendo aderente all'ortografia medio-persiana, non certamente alla sua pronuncia reale. 
Chiarissimo, pertanto, l'intento polemico anti-mazdakita (per quanto trasformato in una veste cristiana con un'esaltazione della verginità), al punto tale che questa storia minore potrebbe aver fatto parte di un repertorio narrativo redatto contro tale eresia iranica. Ricordiamo a tal proposito che doveva circolare ${ }^{60}$ anche una storia secondo la quale Mazdak avrebbe addirittura cercato di giacersi con la madre di Cosroe e che proprio per tale ragione il futuro re gli avesse giurato implacabile vendetta. Infine, anche il fatto che il padre di Koatos, conosciuto il misfatto, lo avrebbe fatto appendere con la testa in basso, nel mezzo della città, affinché fosse divorato dagli uccelli sarcofagi, merita una riflessione. La vicenda, in questo caso non può nuovamente riguardare la persona specifica di Kawād I, ma sembrerebbe comunque echeggiare, mutatis mutandis, la ben nota vicenda della tremenda punizione inflitta da Cosroe I proprio contro i Mazdakiti. Costoro, come sappiamo, sarebbero stati per suo ordine (ancora sotto il governo del padre Kawād) fatti interrare a testa in giù e piedi in alto come in una sorta di bosco degli orrori; ${ }^{61}$ lo stesso Mazdak sarebbe stato impiccato e lasciato appeso. ${ }^{62}$ La dinamica del supplizio non è esattamente la stessa, ma i punti in comune sono diversi. La materia iranica di fondo resta comunque ricca e piena di spunti di riflessione, tutti ambientati alla corte di Kawād I e del suo figlio prediletto Cosroe I e trovano una spiegazione plausibile nella reazione al Mazdakismo, che peraltro aveva suscitato preoccupazioni anche in ambito bizantino. Mi sembra perciò evidente che il carattere scellerato del personaggio di Koatos, figlio di Koatos, presente nella Disputatio, per quanto non corrisponda affatto alla figura storica del sovrano Kawād, si ispiri con ragionevole probabilità ad un repertorio chiaramente ambientato nell'epoca dell'omonimo sovrano persiano. Lo stesso dicasi per il richiamo alla scarnificazione del cadavere del colpevole, evento che, per quanto possa apparire orribile, e tale doveva essere la sua percezione nella sensibilità di un lettore/uditore occidentale, riflette, invece, il normale costume funerario zoroastriano.

Ma gli spunti comparativi non sono affatto finiti. Il tema della morte ritorna, invece, con drammatica ampiezza nella biografia del "secondo" Kawād, ovvero nella storia di Kawād Širōy, šāhān šāh per soli 8 mesi, nel 628; per paura, egli avrebbe lasciato sterminare i suoi 18 fratelli (ed i loro figli), di cui Hamzah $(\S 61)^{63}$ elenca ancora i nomi ${ }^{64} \mathrm{La}$ stessa fonte araba (Hamzah 61.15) ${ }^{65}$ ci informa che il principe Afrūdšāh, uno dei figli di Cosroe II, sarebbe stato fatto assassinare proprio da Kawād II Širōy. ${ }^{66}$ Tale antroponimo appare molto interessante poiché, nella sua varietà, il nome di Frūdšāh/Afarōdšāh, non sarebbe affatto isolato, se confrontato direttamente, almeno per la sua prima parte (ovvero senza il secondo elemento di composizione, $-\check{s} \bar{a} h$ ) con il siriaco $b r w d q$, corrispondente al pahlavi $\bar{A} b r \bar{d} d a g^{67}$ [plwtky]. Si tratta certamente di un derivato di un composto

${ }^{60}$ Tale tradizione è ampiamente diffusa nelle fonti arabo-persiane, ma deve trattarsi di notizia (se leggendario o vera non sappiamo) di età sasanide, che rientra nel complesso repertorio di storie concernenti la vicenda mazdakita; vedi Christensen 1925, 59-60, passim.

61 Yarshater 1983, 994.

${ }^{62}$ Christensen 1925, 60; si veda anche a p. 50.

${ }^{63}$ Cfr. Hamzae Ispahanensis Annalium libri X: vedi Gottwaldt 1844 (testo); 1848, 45 (traduzione).

64 Justi 1895, 297.

${ }^{65}$ Cfr. Hamzae Ispahanensis Annalium libri X; vedi Gottwaldt 1848, 46-47 (traduzione).

${ }^{66}$ Cfr. Justi 1895, 100.

${ }^{67}$ Vedi Gignoux - Jullien - Jullien 2009, 29, n 6a; cfr. Justi 1895, 99. 
formato da $\bar{a} b(<\check{\bar{a}} p-)$ "acqua" + rōd $\left(<*\right.$ rauta-) "fiume", ${ }^{68}$ seguito dal frequentissimo suffisso derivativo -ag $\left(<*_{-} a k a-\right)$. È bene notare che proprio quello di $\bar{A} b r \bar{d} d a g$ fu il nome che sarebbe stato portato in particolare da un eunuco, tra i principali sudditi cristiani di Seleucia al tempo di Cosroe I. ${ }^{69}$ Tale personaggio viene, per esempio, menzionato nel contesto del processo contro Mār Abba ${ }^{70}$ katholikós della Chiesa siro-orientale negli anni 540-552; tale personaggio storico viene, per giunta, indicato come una figura particolarmente autorevole tra i Cristiani di Weh-Ardašīr (Seleucia), e risulta addirittura essere stato in rapporti di amichevole confidenza con lo stesso sovrano, al punto che lo $\check{s} \bar{a} h \bar{a} n \grave{s} \bar{a} h$ in persona sarebbe intervenuto per proteggerlo dalle ripetute accuse rivoltegli contro del clero mazdeo. ${ }^{71}$ Tale notabile cristiano figura anche nel martirio di Mār Yazd-panāh, ove ${ }^{72}$ viene data un'ampia e positiva descrizione del suo profilo personale e del suo tratto oltremodo virtuoso. Hoffmann vorrebbe mettere in connessione diretta con tale prestigioso cristiano della corte di Cosroe una variante onomastica attestata in

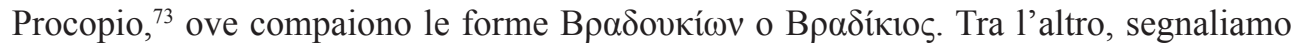
che anche le fonti sfragistiche ci confermerebbero l'esistenza di un personaggio di rango con tale nome, più o meno nello stesso periodo, ma non siamo in grado di affermare se si tratti esattamente della stessa persona o meno. ${ }^{74}$ Ora pare utile notare che, sebbene il

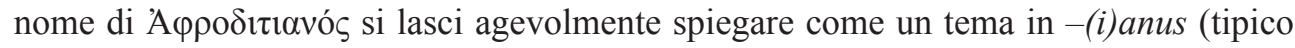
dell'onomastica di epoca tardo-romana), ${ }^{75}$ verisimilmente derivato da un più comune A $\varphi \rho o \delta i ́ \tau 1 o s,{ }^{76}$ a sua volta riconducibile al nome della dea "Afrodite", certamente non può sfuggire la sua somiglianza, anche se del tutto paretimologica, col nome di quello stesso personaggio così famoso presso la corte del sovrano Cosroe, esempio mirabile per giunta di cristiano ed al contempo di amico personale del re. Soprattutto se teniamo in conto le inevitabili oscillazioni, abbastanza frequenti nel consonantismo, tra sorde e sonore, nel passaggio e nell'adattamento dei nomi propri tra medio-persiano e greco, magari attraverso l'ulteriore intermediazione siriaca, il nome di 'A $\rho \rho \delta \imath \tau \imath \alpha v o ́ s$, anziché evocare quello di un lontano governatore egiziano quale quel tale Afrodisius, già men-

68 Vedi Gignoux 1986, $\mathrm{n}^{\circ} 13$.

69 Vedi Gignoux - Jullien - Jullien 2009, 29, n $6 a$.

70 Jullien 2015.

71 Jullien 2015, 20, § 15 .

72 Jullien 2015, xx-xxi, 84, § 10; sulla vicenda di Ābrōdag che compare nel contesto del martirio di Yazdpanāh, si veda anche Hoffmann 1880, 89-90.

73 Vedi ancora Hoffmann 1880, 89-90, nota 810.

74 Vedi Gignoux - Jullien - Jullien 2009, 29, n 6 b.

75 Si deve osservare, come mi fa gentilmente notare il collega ed amico Prof. Velizar Sadovski (Vienna), che proprio il suffisso -(i)anus nell'epoca aurea e argentea è stato il mezzo universale di formazione di: (1.1.) cognomi di persone adottate, che prendono il gentilicium del padre adottivo ed invece ricevono un cognomen dal gentilicium del padre fisico che mettono dopo il nuovo gentilicium: come il figlio di C. Octavius che, dopo l'adozione da parte di Cesare, si chiamava C. Iulius Caesar Octavianus, poi + Augustus. (1.2.) Secondo lo stesso pattern (e per motivi rituali simili) si formano prenomi di liberti dal gentilicium degli ex signori: si tratta in tal caso di formazioni sorte sulla base sia di nomi puramente romani sia di nomi di origine straniera. Sulla moltiplicazione dei nomi in -ianus nel tardo Impero Romano, si vedano in particolare i lavori di Solin 1971; 1990; 2005; 2009; 2012a; 2012b. In particolare sulla diffusione del suffisso -ianus al di fuori del sistema dell'adozione, cfr. Kajanto 1965; Salomies 1987; Tataki 1996, 108.

76 Sulla diffusione di nomi romani nella parte orientale dell'Impero, si rimanda a Dittenberger 1872, 129-155, 281-313; Solin 1994/95; 1990; 2013, 752-753. 
zionato nello Pseudo-Matteo XXIV,1 (che pur avrebbe assistito alla caduta degli idoli in un tempio pagano al momento della comparsa di Gesù e sua madre, come suggerisce la Bringel), ${ }^{77}$ avrebbe potuto richiamare alla memoria un contemporaneo antroponimo di origine propriamente persiana ( $\left.{ }^{*} \bar{A} b r \bar{o} d-(a g)>A \varphi \rho o \delta-\right)$, ben attestato anche in contesto cristiano (vedi siriaco $b r w d q$ ), nel quadro di una correlazione puramente basata su di una somiglianza fonetica e sull'effetto di una (utile) risonanza culturale. Scrivo "utile", perché se l'autore dell'opera voleva richiamare un certo contesto alla memoria di un pubblico che conoscesse sufficientemente la letteratura siriaca e greca di area orientale (ossia pertinente al limes sasanide), la scelta di tale nome poteva inevitabilmente giocare su più registri, interni ed esterni. Ovviamente, anche in questo caso specifico, non si intende affatto asserire che l'Aphroditianus del testo greco sia esattamente l'A $\bar{b}$ rō dag dei martirologi siriaci oppure un altro particolare personaggio storico di Seleucia, ma semplicemente constatare come la scelta narrativa di tale nome potesse trovare un'eco diretta anche nell'onomastica cristiana di Persia e con riferimento a personaggi in posizioni di rilievo tali da risultare come esemplari, segnatamente nel contesto di vicende che risultano sotto molti aspetti evocare l'atmosfera della corte di Cosroe I ed il clima della sua epoca. ${ }^{78}$ Vorrei, peraltro, sottolineare come, alla luce della letteratura siriaca, non fosse affatto raro lo svolgimento di articolate controversie teologiche, spesso di natura cristologica, alla corte sasanide. ${ }^{79}$ Tale fenomeno si ripete ampiamente nel VI e nel VII secolo, e fa da contrappeso alla presenza di polemiche tra Cristiani e Mazdei, ${ }^{80}$ alle quali, invece, il testo greco della Disputatio non fa mai riferimento, probabilmente perché tale soggetto non era strettamente significativo nell'ambito delle finalità apologetiche che si era proposto l'anonimo autore della presente Disputatio. Non dovrà neppure stupire oltremodo l'attenzione verso il mondo iranico di questa particolare opera in greco, soprattutto se consideriamo con attenzione il fatto che la Scuola di Edessa ed i suoi teologi, nonostante le grandi divisioni sul tema dell'economia del complesso teandrico, godevano di profondo rispetto. Personaggi come Mār Abba, una delle menti più illuminate del Cristianesimo di Persia, ebbero modo di predicare in Alessandria d'Egitto, se non addirittura in Grecia, ${ }^{81}$ né si potrà trascurare il fatto che presso la corte costantinopolitana, all'epoca di Giustiniano, esattamente nel 533, sulla scorta della pace tra Romani e Persiani, si tenne un dibattito teologico di ben tre giorni che vide come protagonisti,

${ }^{77}$ Vedi la traduzione inedita on line della Bringel (senza data), nota 3.

${ }^{78}$ Non si deve ignorare che la dimensione intellettuale della controversia teologico-religiosa a corte trova una sua ambientazione anche nel contesto sasanide, con particolare riguardo per le vicende finali dell'eresia mazdakita. Christensen $(1925,124-126)$ ricordava, infatti, come alcune fonti collocassero lo sterminio dei Mazdakiti proprio nel quadro di una disputa tenutasi a corte, alla quale avrebbero partecipato anche sapienti cristiani di alto rango, come naturali alleati, in questo specifico frangente, dei Mazdei. La tradizione relativa allo svolgimento di dibattiti teologici presso la Corte persiana risale però al periodo di Šābuhr I, come sembrerebbe confermato dalla notizie relative alle polemiche tra Mazdei Cristiani e Manichei. Sulla questione si vedano le recenti considerazioni formulate in particolare da Dilley nel recente volume miscellaneo dedicato ai Kephalaia della Chester Beatty Library (Gardner - BeDuhn - Dilley 2015, 15-51, passim).

${ }^{79}$ Vedi in particolare Jullien 2015, xxxix-xlvii (con ulteriore bibliografia).

${ }^{80}$ Vedi ancora Jullien 2015, xlvii-xxvii, passim. Segnalo la recente uscita di un volume miscellaneo, curato da Herman (2014), ed espressamente dedicato alla complessità sociale delle dinamiche interreligiose tra Ebrei, Cristiani e Zoroastriani in ambito sasanide.

${ }^{81}$ Jullien 2015, xxv-liii. 
da una parte, una nutrita e prestigiosa delegazione di vescovi siro-orientali, inviati dallo stesso Cosroe, dall'altra, un importante gruppo di teologi di formazione severiana. ${ }^{82}$ Tutti questi eventi fanno da più ampia cornice alla nostra vicenda e ne accrescono il numero dei motivi di più generale ispirazione.

Ma torniamo per un momento alla figura di Aphroditianus, al quale nell'incipit

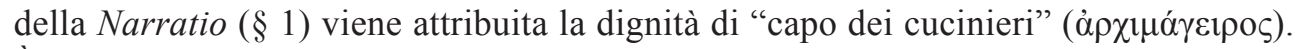
È già stato fatto notare come tale titolo sia di derivazione vetero-testamentaria, essendo utilizzato per Putifar (Gen. 37,36) e Nabuzardan (4 Rg. 25, etc.), ed abbia perciò un'origine molto antica; si tratta, infatti, della traduzione dell'ebraico rab tabahîm, "capo della guardia", ma a partire dal significato di "sgozzare, uccidere" di tabah, che come segnalava la stessa Bringel, ${ }^{83}$ ricalca a sua volta la funzione già babilonese del rab nahatimmu "capo cuciniere", attestato in una lista di dignitari di Nabucodonosor. Sebbene il mondo sasanide non manchi di alti dignitari preposti alla tavola del re ed alla sua cucina, mi domando quanto la scelta di un termine greco di impiego biblico non rifletta il desiderio più o meno cosciente da parte dell'autore di addomesticare una figura chiaramente "aliena", restando Aphroditianus un saggio persiano e verisimilmente mazdeo (almeno nel contesto della Disputatio), senza però chiamarlo

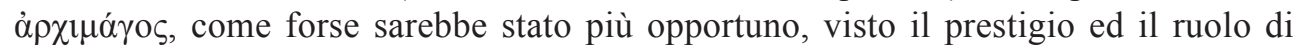
cui tale autorità sembra godere presso il suo re. Si tratta, forse, di un'ulteriore spia di una specifica realtà storica, in cui tale Aphroditianus in realtà non sarebbe affatto stato mazdeo o della volontà specifica di non sovraccaricare positivamente una figura sacerdotale zoroastriana?

In questo breve contributo, intendo toccare un altro soggetto che mi sembra alquanto degno di attenzione. La Bringel ${ }^{84}$ si è giustamente posta la questione delle finalità ${ }^{85}$ dell'opera nella sua veste più o meno completa, per escludere con estrema ragionevolezza che lo scopo prioritario della sua composizione/compilazione fosse quello della conversione degli Ebrei. La nostra Disputatio mostra un'ostilità anti-giudaica ${ }^{86}$ così marcata, almeno rispetto ad altre fonti (ad esempio, la Doctrina Jacobi), da risultare poco efficace, se non addirittura controproducente nel contesto di una progressiva azione di avvicinamento, convincimento didattico e, quindi, di conversione. Più ragionevole mi sembra, invece, la supposizione formulata dalla stessa studiosa francese che in realtà tale narrazione si rivolga, per "uso interno", agli stessi Cristiani, soprattutto per via delle loro spesso troppo faziose controversie. La Bringel ${ }^{87}$ menziona assai opportunamente sia la conclusione dell'opera in cui Aphroditianus, da saggio pagano (ma cristiano in pectore), lancia un invito alla concordia, che non si lascia affatto circoscrivere solo alla rivalità tra Ebrei e Cristiani. Ella, inoltre, sottolinea

82 Jullien 2015, xxix-xxx.

83 Vedi la traduzione inedita on line della Bringel (senza data), nota 1.

${ }^{84}$ Vedi http://ctesiphon.huma-num.fr/sources-litteraires/intro-d/de-gestis-in-perside/\#_ftn73, passim.

${ }^{85}$ Mi sembra utile ricordare però anche la tesi sostenuta da Honigmann (1953, 82-91, in particolare alla p. 86), che reputava l'opera frutto di un intento satirico, per attribuirla addirittura ad uno scrittore pagano di area siriaca. Lo stesso Honigmann (1953, 85 e nota 6) arrivava a postulare la presenza del nome di tale autore, 'Abd-Hadad, in uno dei misteriosi passi in "pseudo-persiano": $\delta 1 \alpha \lambda \alpha \lambda$ ì̀ 'A $\beta \delta \circ \delta \varepsilon \delta \omega ́ \rho o v$.

86 Cfr. anche Külzer 1999.

${ }^{87}$ Vedi http://ctesiphon.huma-num.fr/sources-litteraires/intro-d/de-gestis-in-perside/\#_ftn73, passim. 
l'importanza dell'episodio occorso al $\S 33$, in cui sono addirittura alcuni scaltri e doppi Archimandriti a recarsi presso il re di Persia al fine di calunniare Aphroditianus, colpevole, a loro dire, di avere difeso gli stessi Cristiani. La risposta del sovrano sottolinea ancor di più l'incoerenza e la malevolenza intestina dei Cristiani. Si noterà che in riferimento allo stesso capitolo una glossa conservata nel $\mathrm{ms} \mathrm{O}$, ed opportunamente

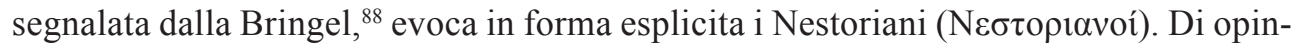
ione simile appare anche la Heyden, ${ }^{89}$ per la quale il nucleo essenziale della Disputatio sarebbe da attribuire ad un cristiano di tendenza alquanto tollerante, il quale ben si collocherebbe in epoca giustinianea. Un altro episodio che mi sembra utile inserire in questo dossier è quello del $\S 16$, in cui è proprio Aphroditianus a sottolineare che, a dispetto della coerenza delle Sacre Scritture, i Cristiani cadono spesso in discredito per via delle loro faziose interpretazioni che li portano a eccessive controversie, spesso incoerenti e prive di una prudente riflessione.

Non è certo questa la sede per aprire una complessa discussione sulle sorti del Cristianesimo nell'Impero sasanide, ma come avevo già sostenuto in altre sedi, ${ }^{90}$ appare evidente come la conflittualità tra confessioni cristiane differenti, soprattutto intorno alle violenti controversie cristologiche, abbia prodotto, in particolare presso la corte persiana, scompigli e danni gravissimi, che emersero in modo palese e devastante durante il regno di Cosroe II. Non si può escludere che certe ammonizioni di Aphroditianus alludano a tali sventurate vicende o che raccolgano, comunque sia, un'espressione di buon senso maturata da qualche autore molto realista e profondamente preoccupato dai pericoli continui che posizioni teologiche inconciliabili andavano producendo nella realtà più spicciola attraverso il ricorso continuo a delazioni, calunnie ed inganni reciproci, che non si esaurivano affatto in uno scontro fratricida, ma che facevano leva proprio sul potere di sovrani non cristiani, come avvenne nel caso paradigmatico dei Sasanidi, al fine di ottenere qualche soluzione favorevole alla propria fazione. Per certi versi, il teatro delle parti messo in scena dalla Disputatio non si discosta molto da una realtà, sicuramente meno nobile di quella letteraria, che ha segnato la storia delle controversie cristiane, soprattutto quelle intestine, alla corte di Persia.

BIBLIOGRAFIA

Bacci, M. (2000), La fisionomia di Cristo nelle testimonianze letterarie del Medioevo, in: G. Morello, G. Wolf (a cura di), Il volto di Cristo, Milano: 33-34.

Blockley, R.C. (1985), The History of Menander the Guardsman. Introductory Essay, Text, Translation, and Historiographical Notes by R.C. Blockley, Liverpool.

Börm, H. (2007), Prokop und die Perser. Untersuchungen zu den römisch-sasanidischen Kontakten in der ausgehenden Spätantike, Stuttgart.

\footnotetext{
${ }^{88}$ Vedi la inedita traduzione on line della Bringel (senza data) alla nota 78 .

89 2009, 158-165 e passim.

${ }^{90}$ Panaino 2004bf.
} 
Bratke, E. (1899), Das sogenannte Religionsgespräch am Hof der Sasaniden, Leipzig.

Bringel, P. (2007), Une polémique religieuse à la cour perse: le De gestis in Perside. Histoire du texte, Edition critique et traduction (Sorbonne, thèse présentée par P. Bringel, sous la direction de J. Gascou), Paris.

Bringel, P. (senza data), Une polémique à la cour perse: le De gestis in Perside. Traduction inédite. http://ctesiphon.huma-num.fr/wp-content/uploads/2015/05/De-Gestis-in-Perside-traduction.pdf.

Christensen, A. (1925), Le règne du roi Kawadh et le communisme Mazdakite, Copenhagen.

Crone, P. (1991), Kavad's Heresy and Mazdak's Revolt, Iran 29: 21-42.

Crone, P. (2014), The Nativist Prophets of Early Islamic Iran: Rural Revolt and Local Zoroastrianism, Cambridge.

Desreumaux, A. (1993), Histoire du roi Abgar et de Jésus. Présentation et traduction du texte syriaque intégral de La Doctrine d'Addaï. En appendice : A. Palmer, Traduction d'une version grecque, et R. Beylot, Traduction d'une version éthiopienne, Turnhout-Paris.

Dittenberger, W. (1872), Römische Namen in griechischen Inschriften und Literaturwerken, Hermes 6: $129-155,281-313$.

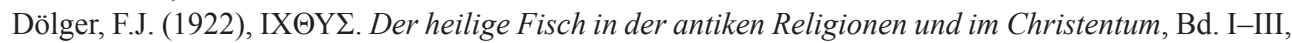
Münster.

Eastbourne, E. (2011), Religious Discussion at the Court of the Sassanids, translated according to the Bratkes' and Bringel's editions, made of public domain on the $19^{\text {th }}$ March 2011, at the website: http://archive.org.

Fiaccadori, G. (2006), Mâsidis (Giovanni di Nikiou, Chron. XC 54-60), in: G. Fiaccadori (a cura di, con la collaborazione di A. Gatti e S. Marotta), «In partibus Clius». Scritti in onore di Giovanni Pugliese Carratelli, Napoli: 113-135.

Frye, R.N. (1983), The Political History of Iran under the Sasanians, in: E. Yarshater (ed.), The Cambridge History of Iran, vol. 3/1: The Seleucid, Parthian and Sasanian Periods, Cambridge: 116-180.

Gardner, I., BeDuhn, J., Dilley, P. (2015), Mani at the Court of the Persian Kings: Studies on the Chester Beatty Kephalaia Codex, Leiden-Boston.

Gariboldi, A. (2009), Il regno di Xusraw dall'anima immortale: riforme economiche e rivolte sociali nell'Iran sasanide del $6^{\circ}$ secolo, 2nda ed., Milano.

Gignoux, Ph. (1986), Noms propres sassanides en moyen-perse épigraphique. (Iranisches Personennamenbuch, Bd. II, Fasc. 2), Wien.

Gignoux, Ph., Jullien, Chr., Jullien, Fl. (2009), Noms propres syriaques d'origine iranienne. (Iranisches Personennamenbuch, Bd. VII, Fasc. 5), Wien.

Gnoli, Gh. (2004), Nuovi studi sul Mazdakismo, in: La Persia e Bisanzio. (Atti dei convegni Lincei 201), Roma: 439-456.

Gottwaldt, J.M.E. (1844-1848), Hamzae Ispahanensis Annalium libri X, edidit J.M.E. Gottwaldt, Lipsiae 1844 (testo) e 1848 (traduzione).

Herman, G. (ed.) (2014), Jews, Christians and Zoroastrians. Religious Dynamics in a Sasanian Context, ed. G. Herman, Piscataway.

Heyden, K. (2006), Die Christliche Geschichte des Philippos von Side: Mit einem kommentierten Katalog der Fragmente, in: M. Wallraff (hrsg.), Julius Africanus und die christliche Weltchronistik, Berlin: 209-243.

Heyden, K. (2009), Die “Erzählung des Aphroditian”. Thema und Variationen einer Legende im Spannungsfeld von Christentum und Heidentum, Tübingen.

Hoffmann, G. (1880), Auszüge aus syrischen Akten persischer Märtyrer übersetzt und durch Untersuchungen zur historischen Topographie erläutert, Leipzig.

Honigmann, E. (1953), Patristic Studies, Città del Vaticano.

Jullien, Chr. (2015), Husraw I ${ }^{\mathrm{er}}$. Reconstruction d'un règne. Sources et documents, textes réunis par Chr. Jullien, Paris.

Jullien, Fl. (2015), Histoire de Mār Abba, Catholicos de L'Orient. Martyres de Mār Grigor, Général en Chef du Roi Khusro I ${ }^{\mathrm{er}}$ et de Mār Yazd-Panāh, Juge et Gouverneur, (Corpus Scriptorum Orientalium 659; Scriptores Syri 255), Lovanii. 
Justi, Fr. (1895), Iranisches Personennamenbuch, Marburg.

Kajanto, I. (1965), Latin Cognomina, Helsinki.

Kampers, Fr. (1901), Alexander der Große und die Idee des Weltimperiums in Prophetie und Sage. Grundlinien, Materialien und Forschungen, Freiburg.

Kaufmann, C.M. (1901), La Pegè du temple de Hierapolis. Contribution à la symbolique du christianisme primitive, $R H R$ 2: 529-548.

Klíma, O. (1957), Mazdak. Geschichte einer sozialen Bewegung im sassanidischen Persien, Praha.

Klíma, O. (1977), Beiträge zur Geschichte des Mazdakismus, Prague.

Külzer, A. (1999), Disputationes Graecae contra Iudaeos. Untersuschungen zur byzantinischen antijüdischen Dialogliteratur und ihrem Judenbild, Stuttgart-Leipzig.

Mayerson, Ph. (1994), Justinian's Novel 103 and the Reorganization of Palestine, in: Ph. Mayerson, Monks, Martyrs, Soldiers and Saracens. Papers on the Near East in late antiquity (1962-1993), Jerusalem: 294-300.

Migne, J.-P. (1856), Patrologia Graecae, t. 10, Lutetiae Parisorum.

Monneret de Villard, U. (1952), Le Leggende Orientali sui Magi Evangelici, Città del Vaticano.

Panaino, A. (2001), L'influsso greco nella letteratura e nella cultura medio-persiana, in: G. Fiaccadori (a cura di), Autori classici in lingue del Vicino e Medio Oriente. Atti del VI, VII e VIII Seminario sul tema: "Recupero di testi classici attraverso recezioni in lingue del Vicino e Medio Oriente" (Milano, 5-6 ottobre 1987; Napoli, 5-6 dicembre 1988; Bologna, 13-14 ottobre 1989), Roma: 29-45.

Panaino, A. (2004a), I Magi evangelici. Storia e simbologia tra Oriente e Occidente, Ravenna.

Panaino, A. (2004b), La Chiesa di Persia e l'Impero Sasanide. Conflitto e Integrazione, in: Cristianità d'Occidente e Cristianità d'Oriente (secoli VI-XI). LI Settimana di Studio della Fondazione CISAM, Spoleto, 24-30 aprile 2003, Spoleto: 765-863.

Panaino, A. (2009), Il duplice volto del protocollo aggiuntivo sulle minoranze religiose nella Pace dei 50 anni, Bizantinistica 11: 273-299.

Panaino, A. (2011), Ciro, i Magi evangelici e la Disputatio De Christo in Persia, Studi Romagnoli 62 (Studi su Ravenna. Studi Vari): 57-73.

Panaino, A. (2011/2012), Christians and Zoroastrians in the Fifty-Years Peace Treaty, Nāme-ye Irān-e Bāstān 11/2: 69-92.

Pourshariati, P. (2008), Decline and Fall of the Sasanian Empire: The Sasanian-Parthian Confederacy and the Arab Conquest of Iran, London-New York.

Salomies, O. (1987), Die römischen Vornamen. Studien zur römischen Namengebung, Helsinki.

Schnetz, J. (1940), Itineraria Romana, Bd. II, Lipsiae.

Schwartz, E. (1894), Aphroditianus, RE 1: 2788-2793.

Solin, H. (1971), Beiträge zur Kenntnis der griechischen Personennamen in Rom, Helsinki.

Solin, H. (1990), Namenpaare. Eine Studie zur römischen Namengebung, Helsinki.

Solin, H. (1994/1995), Anthroponymie und Epigraphik. Einheimische und fremde Bevölkerung, $H y$ perboreus: 109-113.

Solin, H. (2005), Problèmes de l'onomastique du Bas-Empire, in: J.Desmulliez, Christine Hoët-Van Cauwenberghe (éd.), Le monde romain à traves l'épigraphie : méthodes et pratiques, Lille: 271-293.

Solin, H. (2009), Nomi greci nel mondo romano, in: E. Caffarelli, P. Poccetti (a cura di), L'onomastica di Roma. Ventotto secoli di nomi. Atti del Convegno, Roma, 19-21 aprile 2007, Roma: 61-84.

Solin, H. (2012a), Do We Need a New Latin Onomasticon?, in: T. Meißner (ed.), Personal Names in the Western Roman World: Proceedings of a Workshop Convened by Torsten Meißner, José Garcia Ramón and Paolo Poccetti, held at Pembroke College, Cambridge, 16-18 September 2011, Berlin: 3-9.

Solin, H. (2012b), Griechentum in Rom im Spiegel der Namengebung, in: L.-M. Günther, V. Grieb (hrsg.), Das imperiale Rom und der hellenistische Osten. Festschrift für Jürgen Deininger zum 75. Geburtstag, Stuttgart: 89-99.

Solin, H. (2013), s.v. Name, in: Reallexikon für Antike und Christentum 25: 729-795. 
Tataki, A.B. (1996), The nomina of Macedonia, in: A.D. Rizakis (ed.), Roman Onomastics in the Greek East. Social and Political Aspects. Proceedings of the International Colloquium Organized by the Finnish Institute and the Centre for Greek and Roman Antiquity, Athens 7-9 September 1993, (ME $\Lambda$ ETHMATA 21), Athens-Paris: 105-109.

Wiesehöfer, J, (2009), Kawad, Khusro I and the Mazdakites. A new proposal, in: Ph. Gignoux et al. (éd.), Trésors d'Orient, Paris: 391-409.

Wirth, A. (1894), Aus orientalischen Chroniken, Frankfurt am Main.

Yarshater, E. (1983), Mazdakism, in: E. Yarshater (ed.) Cambridge History of Iran: The Seleucid, Parthian and Sasanian Periods, vol. 3/2, Cambridge: 991-1024. 